Meta

Journal des tradlucteurs

Translators' Journal

\title{
Sans ménagement pour « management »
}

\section{Robert Dubuc}

Volume 15, numéro 2, juin 1970

URI : https://id.erudit.org/iderudit/002617ar

DOI : https://doi.org/10.7202/002617ar

Aller au sommaire du numéro

Éditeur(s)

Les Presses de l'Université de Montréal

ISSN

0026-0452 (imprimé)

1492-1421 (numérique)

Découvrir la revue

Citer cet article

Dubuc, R. (1970). Sans ménagement pour « management ». Meta, 15(2),

110-112. https://doi.org/10.7202/002617ar d'utilisation que vous pouvez consulter en ligne.

https://apropos.erudit.org/fr/usagers/politique-dutilisation/ 


\section{PROBLÈMES ET SOLUTIONS}

\section{SANS MÉNAGEMENT POUR "MANAGEMENT"}

Dans un communiqué récent, l'Académie française nous apprenait que le mot "management » faisait désormais partie de la grande famille de la langue française ${ }^{1}$. Ce qui étonne dans cette légitimation d'un emprunt pour le moins discutable, c'est l'absence totale de justification : on n'a même pas cru nécessaire de définir l'acception du terme ainsi proposé à l'usage.

Cette question pose un problème intéressant de terminologie auquel il convient de s'arrêter, toute révérence gardée pour les augustes académiciens. Il faut d'abord établir la notion que recouvre le terme anglais management. Le terme anglais est d'une compréhension assez vaste et s'applique tout autant à l'aspect pratique qu'à l'aspect théorique de cette technique. Il désigne en outre les personnes qui sont responsables du fonctionnement de l'entreprise ${ }^{2}$. Il ne semble pas qu'il y ait en français un mot unique pour désigner ces trois réalités.

Le mot gestion est assez répandu dans l'usage actuel comme équivalent de management au sens concret, c'est-à-dire pour désigner la direction effective de toutes les activités nécessaires pour atteindre les objectifs de l'entreprise. C'est d'ailleurs sur cette définition que repose la distinction faite par le Grand Larousse encyclopédique et l'Aide-mémoire Dunod entre organisation et gestion. «Dans les services administratifs, la gestion s'oppose à l'organisation qui est l'étude des méthodes de gestion ${ }^{3}$. " "L'organisation est à la gestion ce que la statique est à la dynamique. Les organisateurs définissent et mettent en place les structures,

1. Dans son communiqué du 27 février 1969, l'Académie française a proposé comme équivalent du mot anglais management, le mot «management» prononcé à la française (Défense de la langue française, juin 1969, p. 7 ).

2. "Management includes direction of all the activities necessary to achieve the objectives of an enterprise. Management utilizes a multiplicity of principles and practices to develop a consistent way to accomplish desired results." (A.J. Lindemann, E.F. Lundgre et H.K. von Kaas, Dictionary of Management Terms, Dubuque (Iowa), Wm. C. Brown Book Company, 1966, p. 47).

3. Grand Larousse encyclopédique, Paris, Larousse, 1960, 10 vol., au mot gestion. 
les méthodes et les processus que les gestionnaires mettent en œuvre ${ }^{4} . \gg \mathrm{Il}$ semble bien qu'on tienne ici les deux équivalents qui recouvrent le mieux les deux aspects, théorique et pratique, du management. Si l'on veut une confirmation supplémentaire, on peut faire appel au témoignage de Jean Chevalier : «L'organisation du travail en tant que science, écrit-il, ...est une philosophie de l'activité créatrice qui dégage de l'expérience les règles de l'utilisation la plus efficace du travail et des capitaux mis en ouvre; elle est aussi une économie qui vise à harmoniser les facteurs de la production et à ajuster la production et la consommation, c'est-à-dire les moyens d'action et le résultat en vue duquel ils ont été conjugués ${ }^{5}$.

$\mathrm{Si}$ organisation couvre l'aspect théorique du management et gestion, l'aspect pratique, il reste à trouver un équivalent pour le troisième sens : dirigeants qui collectivement assument la gestion et l'organisation de l'entreprise. Le mot direction semble s'imposer ici d'emblée. Les définitions qu'en donnent le Dictionnaire Robert et le Grand Larousse encyclopédique sont explicites et suffisent à justifier l'équivalence.

On voit donc qu'il est possible en français, même en tenant compte de l'usage des spécialistes francophones, de trouver à management des équivalents acceptables. Pourtant l'Académie a donné sa caution à l'emprunt «management». Cette décision des Immortels semble assez révélatrice du fixisme sémantique du français au $\mathrm{Xx}^{e}$ siècle, en particulier quand il s'agit de notions qui viennent au français par l'intermédiaire d'une langue étrangère. Aucun vocable français ne semble alors adéquat. On se refuse à donner aux mots du fonds français le petit coup de pouce qui en fera évoluer le sens pour l'adapter à la réalité nouvelle à désigner. On préfère plutôt accréditer l'emprunt. Il ne s'agit pas de contester la validité de l'ernprunt comme moyen d'enrichir un idiome. L'histoire des langues atteste trop le rôle de l'emprunt pour qu'on puisse l'écarter de façon systématique.

Par contre, lorsqu'une langue emprunte sans nécessité, elle présente des symptômes de faiblesse. Ce faisant, elle renonce à sa conception propre de la réalité. Sur un point de détail, cela importe assez peu. Mais sur un ensemble considérable de termes, une telle démission peut finir par avoir des répercussions assez graves sur la nature même de la langue emprunteuse.

La question n'est pas que «management » soit un emprunt en soi condamnable. Chevalier en fait remonter l'origine au vieux mot français ménagement qui désignait proprement l'art de conduire les affaires ${ }^{6}$. Mais le problème se situe à un autre niveau. L'emprunt est tout simplement inutile. "Management» ne dit rien de plus que gestion et organisation. Pourquoi alors vouloir à tout prix leur substituer un emprunt? On objectera peut-être que «management» pourrait avoir une valeur générique qui fait défaut à gestion et à organisation. Or, précisément, le Grand Larousse encyclopédique donne à «management » le sens suivant : "Science de la technique de direction et de gestion de l'entreprise ${ }^{7} \gg$, définition qui équivaut à celle que Chevalier donne de l'organisation. Il n'a donc pas de valeur générique.

Il est certain que les techniques de gestion ne sont plus ce qu'elles étaient

4. Jean Gerbier, Organisation-Gestion, Paris, Dunod, "Aide-mémoire », 1964, vol. I, préface, p. V.

5. Jean Chevalier, Organisation, $10^{\mathrm{e}}$ éd., Paris, Dunod, 1961, vol. I, p. 39.

6. lbid., p. 58.

7. Supplément, au mot management. 
au XIX siècle. Mais s'il fallait changer d'appellation à chaque mutation que subit un concept, la langue serait constamment à refaire. L'instinct populaire est beaucoup plus sain, lui qui n'hésite pas à continuer d'appeler ferret le bout du lacet même si les fabricants ont substitué depuis longtemps le plastique au métal.

ROBERT DUBUC

1. L. Brunat, Lamy Transport, tome I, Paris, Services Lamy, 1969, p. 153. 